\title{
Normalizers and split extensions
}

\author{
Dominique Bourn and James R.A. Gray
}

\begin{abstract}
We make explicit a larger structural phenomenon hidden behind the existence of normalizers in terms of existence of certain cartesian maps related to the kernel functor.
\end{abstract}

\section{Introduction}

Any basic textbook on Algebra, see for instance [19, defines as early as in the first pages what is the normalizer of a subgroup $H \subset G$, and this would suggest that this construction is of some consequence. But, beyond a few loose connections with the notion of centralizers, it is not really used and worked out. On the contrary, we shall show here that, in a very conceptual way, the existence of normalizers has two strong structural consequences.

The second author introduced in [17] an abstract notion of normalizer in a pointed category $\mathbb{C}$ as a universal decomposition $U \stackrel{u}{\longmapsto} N \stackrel{w}{\longmapsto} T$ of the monomorphism $U \longmapsto T$ with $u$ a normal monomorphism. We shall investigate here a slightly stricter definition, dropping the condition that $w$ is a monomorphism, which in the category $G p$ of groups is equivalent to the previous one. So that the category $G p$ has normalizers in this sense, and this is equally the case for the category $R g$ of non commutative (non unitary) rings and $R$-Lie of Lie algebras on a ring $R$. When a category $\mathbb{E}$ is cartesian closed, the category $G p \mathbb{E}$ of internal groups in $\mathbb{E}$ has normalizers as well.

Not only this new approach forces, in any case, the second part $w$ of the universal decomposition to be necessarily a monomorphism, but it is hiding a larger structural phenomenon concerning split extensions. In order to enter into that, let us start with a pointed category $\mathbb{C}$, let us denote by $K P t \mathbb{C}$ the category whose objects are the split extensions in $\mathbb{C}$ :

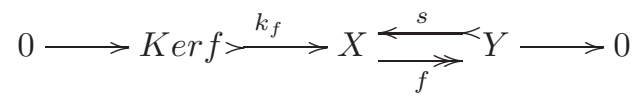

and morphisms are the natural morphisms between split extensions, and by $K: K P t \mathbb{C} \rightarrow \mathbb{C}$ the kernel functor. We shall show here that $\mathbb{C}$ has normalizers if and only if this functor $K$ is fibrant on monomorphisms, namely if and only if it has cartesian maps above any monomorphism.

This last property has two major structural consequences at the level of the fibrations of points $P_{\mathbb{C}}: P t \mathbb{C} \rightarrow \mathbb{C}$, where $P t \mathbb{C}$ is the category whose objects are 
the split epimorphisms and morphisms the commutative squares between them, and where $\mathrm{P}_{\mathbb{C}}$ is the codomain functor, when the category $\mathbb{C}$ is protomodular, namely when the change of base functors with respect to this fibration are conservative [7]. First the category $\mathbb{C}$ is action accessible [10], i.e. satisfies a certain classification property for the split epimorphisms with a given kernel, see Section 3. Secondly the category $\mathbb{C}$ is fiberwise algebraically cartesian closed [15], [16], 9, namely any change of base functor with respect to the fibration of points along a split epimorphism admits a right adjoint.

This article is organized along the following lines:

Section 1 is devoted to the definition, the first examples of normalizers and the characterization in terms of $K$-cartesian maps; Section 2 to the special case of internal algebraic structures; Section 3 to the relationship with action accessibility and Section 4 to the relationship with algebraic cartesian closedness and fiberwise algebraic cartesian closedness.

\section{Normal subobjects and normalizers}

\section{$1.1 \quad$ Normalizers}

Let $\mathbb{E}$ be a finitely complete category. Let $u: U \longmapsto X$ be a monomorphism and $R$ an equivalence relation on $X$. Let us recall from [4] the following:

Definition 1.1. The monomorphism $u$ in $\mathbb{E}$ is said to be normal to $R$ if: i) we have: $u^{-1}(R)=\nabla_{U}$;

ii) the induced internal functor

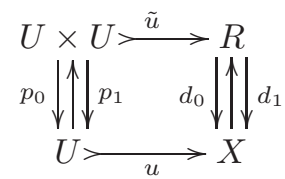

is a discrete fibration.

When $\mathbb{E}$ is the category of sets, an inclusion map $U \longmapsto X$ is normal to an equivalence class of $R$ on $X$ in this sense if and only if $U$ is either empty or is one of the equivalence classes of $R$. In particular the inclusion $\emptyset \longmapsto X$ is normal to every equivalence relation on $X$. When $\mathbb{E}$ is pointed, any map has a kernel, and the kernel $k_{h}: K[h] \longmapsto X$ of any map $h: X \rightarrow Y$ is normal to the kernel equivalence relation $R[h]$ of the map $h$. On the other hand, given an equivalence relation $d_{0}, d_{1}: R \rightrightarrows X$ the pullback diagram

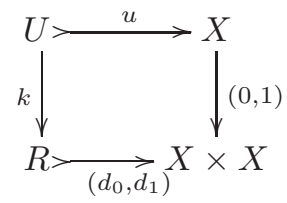


defines the normalization $u: U \rightarrow R$ of $R$, which is automatically normal to $R$ (see [4). Furthermore if $u: U \longmapsto X$ is normal to $R$, then there exists $k: U \rightarrow R$ such that (10) is a pullback. Now let $v: U \longmapsto T$ be any monomorphism in $\mathbb{E}$.

Definition 1.2. We say that $v$ has a normalizer when there is a pair $\left(u, R_{v}\right)$ with $u: U \longmapsto X$ normal to $R_{v}$ and factorization $w: X \rightarrow T$ such that $v=w \cdot u$ which is universal with respect to this kind of specific decomposition of $v$. We say that $\mathbb{E}$ has normalizers when any monomorphism $v$ has a normalizer.

Proposition 1.3. Suppose that $E$ is pointed and $v$ has a normalizer; then the factorization $w$ is necessarily a monomorphism.

Proof. Let us consider the kernel equivalence relation of $w$ :

$$
R[w] \underset{p_{1}}{\stackrel{p_{0}}{\rightleftarrows}} X \underset{w}{\longrightarrow} T
$$

We define the equivalence relation $R_{v} \square R[w]$ on the object $R[w]$ as the inverse image of $\left(R_{v} \times R_{v}\right)$ along $\left(p_{0}, p_{1}\right): R[w] \longmapsto X \times X$. Accordingly (see [4), its normalization is the pullback of $v \times v: U \times U \longmapsto T \times T$ along this same map $\left(p_{0}, p_{1}\right)$, namely the factorization $(u, u): U \longmapsto R[w]$. According to the universal property of $\left(u, R_{v}\right)$, there is a unique factorization of the map $w \cdot p_{0}$ through $X$; thus we get $p_{1}=p_{0}$ and $w$ is a monomorphism.

Examples. It is easy to check that the pointed categories $G p$ of groups, $R g$ of non commutative non unitary rings and $R$-Lie of Lie algebras on a ring $R$ have normalizers in this sense.

\subsection{Normalizers and split extensions}

From now on, we shall suppose that the category $\mathbb{E}$ is pointed. As usual we denote by $P t \mathbb{E}$ the category whose objects are the split epimorphisms in $\mathbb{E}$ with a given splitting, and maps those pairs of maps between these data such that the squares obtained by considering the split epimorphisms and the splittings alone commute, and by $\mathrm{P}_{\mathbb{E}}: P t \mathbb{E} \rightarrow \mathbb{E}$ the functor associating with any split epimorphism its codomain, which is a fibration whose $\mathrm{P}_{\mathbb{E}}$-cartesian maps are the pullbacks of split epimorphisms. When $\mathbb{E}$ is pointed, we shall denote by $K P t \mathbb{E}$ the category of split extensions, namely of split epimorphisms with a chosen kernel, and by $K: K P t \mathbb{E} \rightarrow \mathbb{E}$ the functor associating with any split extension the domain of its kernel. The functor $K$ is not only left exact, but it creates pullbacks and equalizers. On the other hand, it is clear that the forgetful functor $H: K P t \mathbb{E} \rightarrow P t \mathbb{E}$ is a fully faithful and essentially surjective, namely that it determines a weak equivalence of categories, making the functor $\mathrm{P}_{\mathbb{E}} \circ H$ a split fibration. On the other hand, the functor $K$ has a canonical section $J: \mathbb{E} \rightarrow K P t \mathbb{E}$ associating with any object $T$ the following split extension:

$$
T \stackrel{\left(0,1_{T}\right)}{\longrightarrow} T \times T \underset{s_{0}^{T}}{\stackrel{p_{0}^{T}}{\longrightarrow}} T
$$


Proposition 1.4. Let $v: U \longmapsto T$ be any monomorphism in $\mathbb{E}$. Then $v$ has a normalizer if and only if the map $v: U \longmapsto T=K J(T)$ admits a $K$-cartesian map above it.

Proof. Suppose $v$ has a normalizer $\left(u, R_{v}\right)$ we are going to show that the following right hand side map $(w, \tilde{w}, v)$ (with $\left.\tilde{w}=\left(w \cdot d_{0}, w \cdot d_{1}\right)\right)$ in $K P t \mathbb{E}$ is a $K$-cartesian map above $v$ :

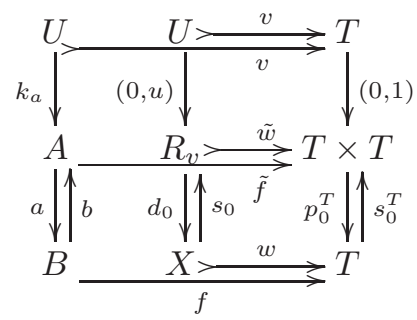

So, suppose we have a map $(f, \tilde{f}, v)$ between the above extremal split extensions of $\mathbb{E}$; we have to find a factorization in $K P t \mathbb{E}$ between the two left hand side split extensions which induces $1_{U}$ at the level of kernels. The map $\tilde{f}$ is necessarily of the form $(f \circ a, t)$ for some $t: A \rightarrow T$ satisfying $t \circ b=f$. Moreover the commutation at the upper level means that $t \circ k_{a}=v$ where $k_{a}$ is normal to $R[a]$. The universal property of the normalizer determines a factorization $\tau: A \rightarrow X$ such that $w \circ \tau=t, \tau \circ k_{a}=u$ and $\tau$ is underlying a map of equivalence relation:

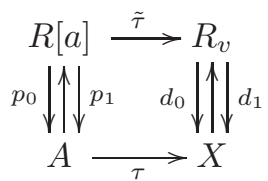

The factorization required for a $K$-cartesian map will be given by:

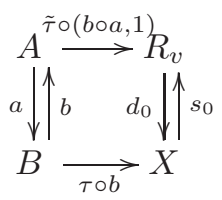

since we have: $w \circ \tau \circ b=t \circ b=f$ and $\tilde{w} \circ \tilde{\tau} \circ(b \circ a, 1)=(f \circ a, t)$ by composition with $p_{0}^{T}$ and $p_{1}^{T}$; moreover we get $\tilde{\tau} \circ(b \circ a, 1) \circ k_{a}=(0, u)$ by composition by the monomorphism $\tilde{w}$ which implies that this factorization induces $1_{U}$ at the level of kernels.

We have now to prove the converse. First let us observe that any $K$-cartesian map above a monomorphism is necessarily a monomorphism. It is a consequence of the fact that the functor $K$ creates pullbacks; for that consider the following 
right hand side $K$-cartesian map of split extensions:

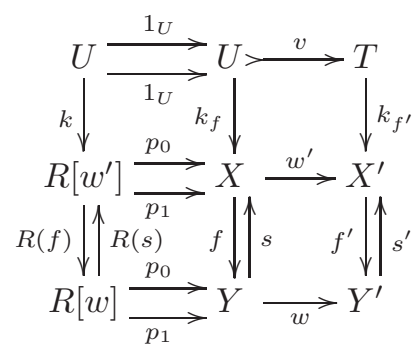

Complete it with the kernel relations of the horizontal maps; this determines the unique left hand side vertical split extension. The uniqueness of the factorization through the $K$-cartesian map $\left(w, w^{\prime}, v\right)$ in $K P t \mathbb{E}$ implies that $p_{0}=p_{1}$ at the two levels, and accordingly that $w$ and $w^{\prime}$ are monomorphisms.

Now suppose that $v: U \longmapsto T=K J(T)$ admits a $K$-cartesian map above it:

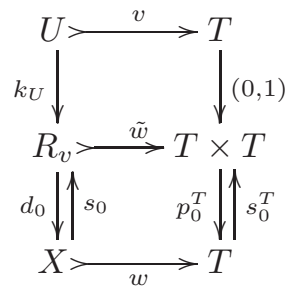

The map $\tilde{w}$ is necessarily of the form $\left(w \circ d_{0}, \delta_{1}\right)$ with $\delta_{1}: R_{v} \rightarrow T$ such that $\delta_{1} \circ s_{0}=w$ and $\delta_{1} \circ k_{U}=v$. Let us show that $\delta_{1}$ factors through $w$ by a (unique) map $d_{1}: R_{v} \rightarrow X$; then, since $w$ and $\tilde{w}$ are monomorphisms, the pair $\left(d_{0}, d_{1}\right): R_{v} \rightrightarrows X$ will be a relation, actually a reflexive relation since, from $w \circ d_{1} \circ s_{0}=\delta_{1} \circ s_{0}=w$, we shall get $d_{1} \circ s_{0}=1_{X}$. For that, let us consider the following diagram of vertical split extensions:

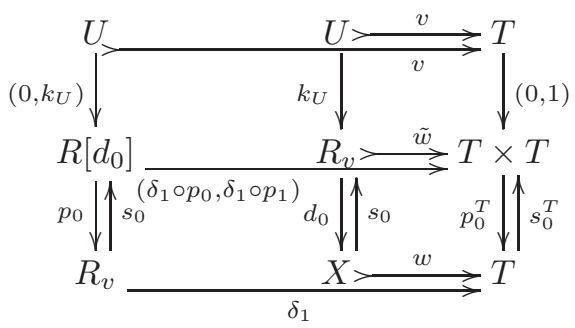


Since we have a $K$-cartesian map, we get a factorization:

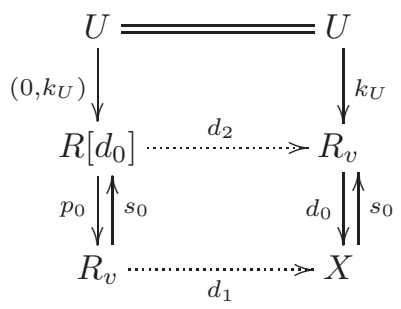

such that $w \circ d_{1}=\delta_{1}$ and $\tilde{w} \circ d_{2}=\left(\delta_{1} \circ p_{0}, \delta_{1} \circ p_{1}\right)$, namely $w \circ d_{0} \circ d_{2}=\delta_{1} \circ p_{0}$ and $\delta_{1} \circ d_{2}=\delta_{1} \circ p_{1}$; and since $w$ is a monomorphism, we get $d_{0} \circ d_{2}=d_{1} \circ p_{0}$ and $d_{1} \circ d_{2}=d_{1} \circ p_{1}$. These equalities on $d_{2}$ show that $\left(d_{0}, d_{1}\right): R_{v} \rightrightarrows X$ is actually an equivalence relation on $X$. Finally, since $k_{U}$ is the kernel of $d_{0}$, the monomorphism $u=d_{1} \circ k_{U}: U \longmapsto X$ is normal to $R_{v}$, and $w \circ u=w \circ d_{1} \circ k_{U}=$ $\delta_{1} \circ k_{U}=v$.

It remains to show that $\left(u, R_{v}\right)$ has the universal property of a normalizer. Let $v=h \circ u^{\prime}$, with $u^{\prime}$ normal to $S$ another decomposition of $v$. Let us consider the following diagram of split extensions with $\tilde{h}=\left(h \circ d_{0}, h \circ d_{1}\right)$ :

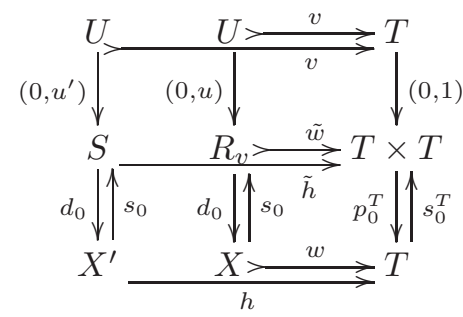

According to the universal property of a $K$-cartesian map, we get a factorization:

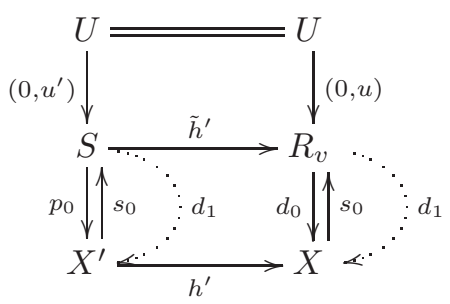

such that $w \circ h^{\prime}=h$ and $\tilde{w} \circ \tilde{h}^{\prime}=\tilde{h}$. From that we get $w \circ d_{1} \circ \tilde{h}^{\prime}=p_{1}^{T} \circ \tilde{w} \circ \tilde{h}^{\prime}=$ $p_{1}^{T} \circ \tilde{h}=h \circ d_{1}=w \circ h^{\prime} \circ d_{1}$; accordingly $d_{1} \circ \tilde{h}^{\prime}=h^{\prime} \circ d_{1}$, and $h^{\prime}$ determines a map $S \rightarrow R_{v}$ of equivalence relation, as desired.

The following observation which is completely invisible at the level of normalizers, will be needed later on:

Proposition 1.5. Let $\mathbb{E}$ be a finitely complete category. Then the $K$-cartesian monomorphisms are stable under pullbacks along $\mathrm{P}_{\mathbb{E}}$-cartesian maps. 
Proof. Let $\left(m, \mu_{X}, \mu_{Y}\right)$ be a $K$-cartesian map and let $\left(m, \nu_{X^{\prime}}, \nu_{Y^{\prime}}\right)$ be the map in $K P t \mathbb{E}$ obtained by pulling back $\left(m, \mu_{X}, \mu_{Y}\right)$ along $\left(1_{K[f]}, x, y\right)$, where $(y, x)$

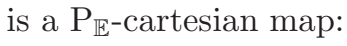

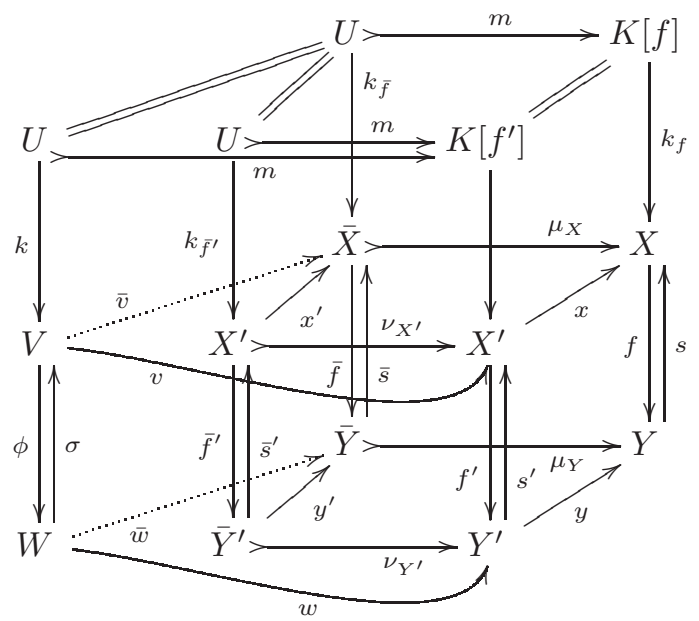

Now let $(m, v, w)$ be a map in $K P t \mathbb{E}$. The map $(m, x \circ v, y \circ w)$ determines a factorization $\left(1_{U}, \bar{v}, \bar{w}\right)$ through the $K$-cartesian map $\left(m, \mu_{X}, \mu_{Y}\right)$ which itself produces the desired factorization through the pullback $\left(m, \nu_{X^{\prime}}, \nu_{Y^{\prime}}\right)$ of $\left(m, \mu_{X}, \mu_{Y}\right)$.

\subsection{Some examples of normalizers}

\subsubsection{Normal subobject and normalizer}

In the category $G p$ of groups, it is clear that if a normal subobject $u$ is normal to an equivalence relation $R$, then its normalizer is $(u, R)$. More generally we get the following:

Proposition 1.6. Let $\mathbb{C}$ be a pointed category. The following are equivalent:

(a) For each reflexive relation $R$ on $X$, the map of split extensions

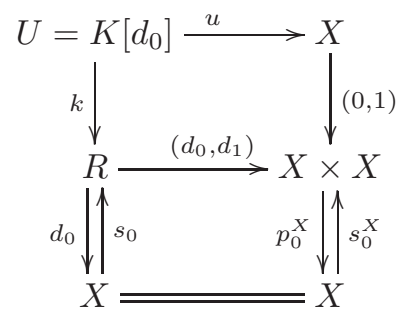

is $K$-cartesian;

(b) $\mathbb{C}$ is Mal'tsev [8, 9] and every normal monomorphism is normal to exactly one equivalence relation. 
Proof. $(b) \Rightarrow(a)$ Since $\mathbb{C}$ is a Mal'tsev category the reflexive relation $R$ is an equivalence relation and $u$ is normal to $R$. Let us show that it coincides with its normalizer. Suppose that $u=h \circ v: U \longmapsto T \rightarrow X$ with $v$ normal to $S$. The unique possible factorization is obviously $h$; so we have to show that $h$ determines a map of equivalence relations $S \rightarrow R$. Since $\mathbb{C}$ is Mal'tsev the relation $R^{\prime}$ defined by the pullback

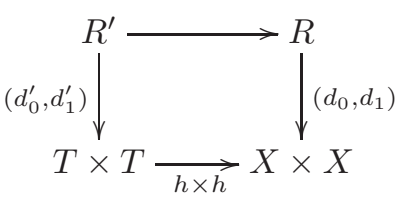

is an equivalence relation. It is easy to check that there is a map $U \times U \rightarrow S \cap R^{\prime}$ such that the square at the top of the diagram

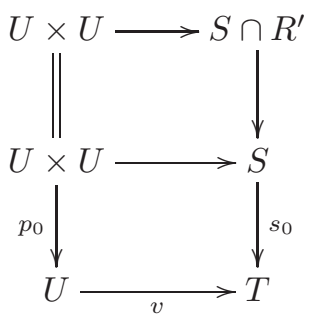

commutes and is therefore a pullback. It follows that the composite of the two squares in the diagram above is a pullback, and therefore that $v$ is normal to $S \cap R^{\prime}$. It follows by assumption that the inclusion $S \cap R^{\prime} \subset S$ is an isomorphism. The composite $S \cong S \cap R^{\prime} \rightarrow R^{\prime} \rightarrow R$ gives the desired factorization.

$(a) \Rightarrow(b)$ The proof of Proposition 1.4 shows that $R$ is actually an equivalence relation and, accordingly, that $\mathbb{C}$ is a Mal'tsev category. Moreover, if two equivalence relations $R$ and $\bar{R}$ has the same normalization $u$, then the two associated $K$-cartesian maps above $u$ show that the two equivalence relations $R$ and $\bar{R}$ are isomorphic.

Remark 1.7. Since every pointed protomodular category is Mal'tsev and by Theorem 6 in [4] every normal monomorphism is normal to exactly one equivalence relation it follows from Proposition 1.6 that Condition (a) of Proposition 1.6 holds in every pointed protomodular category, which can also be proved easily using Lemma 5.1 of [8].

Recall a paragroup [11] is a set $X$ equipped with a binary operation satisfying the cancellation rule $(y / x) /(z / x)=(y / z)$ as well as $x /(x / x)=x$ and $x / x=y / y$. A non empty paragroup $X$ determines a group structure defined by $x / x=1$ and $x . y=x /(1 / y)$. Accordingly the variety Pgr of paragroups is obtained by adding the empty set to the category of groups. It is no longer a protomodular category, but still is a Mal'tsev category. Besides the group monomorphisms, the only other monomorphisms are the initial maps $\alpha_{X}: \emptyset \longmapsto X$ which are all 
normal to any equivalence relation $R$ on $X$. But the normalizer of this map is the pair $\left(\alpha_{X}, \nabla_{X}\right)$ where $\nabla_{X}$ is the indiscrete equivalence relation. Accordingly $P g r$ is a (non-pointed) example of a Mal'tsev category with normalizers in which a normal monomorphisms $u$ can be normal to an equivalence relation $R$, but $(u, R)$ is not the normalizer of $u$.

\subsubsection{A glance at topological groups}

Recall from [5]:

Proposition 1.8. A subobject $A \longmapsto B$ is normal in the category of topological groups if and only if:

1. forgetting the topological structure $A$ is a normal subgroup of $B$;

2. the map $\phi: A \times B \rightarrow A ;(a, b) \mapsto b^{-1} a b$ is continuous as a map of topological spaces.

Next we give an alternative description of a normal subobject.

Lemma 1.9. A subobject $A \longmapsto B$ is normal in the category of topological groups if and only if for each open set $U$ of $A$

(a) for each $b$ in $B$ the set $b U b^{-1}$ is an open subset of $A$;

(b) for each a in $U$ there exists $V$ open in $B$ and $U_{a}$ open in $A$ such that:

(i) 1 is in $V$;

(ii) $a$ is in $U_{a}$;

(iii) for each $b \in V b^{-1} U_{a} b$ is contained in $U$.

Proof. Suppose $A \longmapsto B$ is normal. Let $b$ be an element of $B$ and let $U$ be an open set of $A$. It easily follows from Proposition 1.8 that for each $b$ the map $A \rightarrow A a \mapsto b^{-1} a b$ is continuous and so the set $b U b^{-1}$ is open in $A$. Since $\phi$ is continuous $\phi^{-1}(U)$ is open in $A \times B$ we have for each $a$ in $U$ that $(a, 1)$ is in $\phi^{-1}(U)$, it follows that there exist open sets $U_{a}$ and $V$, of $A$ and $B$ respectively, such that $a \in U_{a}, 1 \in V$ and $U_{a} \times V$ is in $\phi^{-1}(U)$. It follows that for each $b$ in $V, b^{-1} U_{a} b$ is a subset of $U$ as required. Conversely, clearly condition (a) applied to the open set $A$ proves that $A$ considered as a subgroup of $B$ is normal. Let $U$ be an open set of $A$. For each $\left(a^{\prime}, b^{\prime}\right) \in \phi^{-1}(U)$ we have by (b) for $a=\left(b^{\prime}\right)^{-1} a^{\prime} b^{\prime}$ in $U$ that there exists $V$ open in $B$ and $U_{a}$ open in $A$ such that:

(i) 1 is in $V$;

(ii) $a$ is in $U_{a}$;

(iii) for each $b \in V b^{-1} U_{a} b$ is contained in $U$.

The proof is completed by checking that $\left(a^{\prime}, b^{\prime}\right) \in\left(b^{\prime} U_{a}\left(b^{\prime}\right)^{-1}\right) \times\left(b^{\prime} V\right) \subseteq \phi^{-1}(U)$. 
Proposition 1.10. The normalizer of a subobject $A \longmapsto B$ in the category of topological groups exists if the condition (b) of Lemma 1.9 holds. Moreover, under this condition it is defined by

$$
N=\left\{b \in B \mid \forall U \text { open in } A \text {, the sets } b U b^{-1} \text { and } b^{-1} U b \text { are open in } A\right\}
$$

with the topology induced by $B$.

Proof. It follows from Lemma 1.9 that we only need to show that $N$ is a subgroup of $B$, which is easy.

The following example shows that the normalizer of a monomorphism may exist even if Condition (b) of Lemma 1.1 does not hold, moreover the topology of the normalizer $A \rightarrow B$ may not be induced by the topology on $B$ :

Example 1.11. Since the category of topological groups is a regular category [2] and since in regular Mal'tsev categories normal monomorphisms are stable under regular images [7] it follows that we need only show that the normalizer of monomorphism is terminal amongst those factorizations where the second morphism is a monomorphism. Let $G$ be the free group on $\{x, y\}$, and let $G_{d}$ and $G_{i}$ be $G$ with the discrete and indiscrete topology respectively. We will show that the normalizer of the inclusion of $G_{d}$ in $G_{i}$ exists and is $G_{d}$. Suppose $G_{d}$ is normal in $G_{t}$ (a topological group with underlying group $G$ ). It is easy to show that only the elements in $\langle x\rangle$ (the subgroup generated by $x$ ) commute with $x$, and similarly only the elements $\langle y\rangle$ commute with $y$. It follows from Condition (b) of Lemma 1.9 applied to the open sets $\{x\}$ and $\{y\}$, that there exist open sets $V_{x}, V_{y}$ in $G_{t}$ and $U_{x}, U_{y}$ in $G_{d}$ such that:

(i) 1 is in $V_{x}$ and $V_{y}$;

(ii) $x$ is in $U_{x}$ and $y$ is in $U_{y}$;

(iii) for all $b \in V_{x} b^{-1} U_{x} b$ is contained in $\{x\}$ and for all $b \in V_{y} b^{-1} U_{y} b$ is contained in $\{y\}$.

We see that $U_{x}=\{x\}$ and $U_{y}=\{y\}$, and $V_{x} \subset\langle x\rangle$ and $V_{y} \subset\langle y\rangle$, and therefore that $\{1\}=V_{x} \cap V_{y}$ is open, and so $G_{t}=G_{d}$.

\subsection{A characterization}

The next step will consist in showing that the existence of normalizers in $\mathbb{E}$ is equivalent to the fact that the functor $K$ is fibrant on monomorphisms, namely to the fact that there are $K$-cartesian maps above any monomorphism in $\mathbb{E}$.

Lemma 1.12. Let $U: \mathbb{F} \rightarrow \mathbb{E}$ be any left exact functor creating pullbacks. Let $v: Y \longmapsto X$ be a $U$-cartesian monomorphism in $\mathbb{F}$ and $t: X^{\prime} \longmapsto X$ any monomorphism such that $U(v)$ factors through $U(t)$, by means of a map $w$. Then there exist a $U$-cartesian map above $w$. 
Proof. Since $U$ creates pullbacks, there exists a pullback diagram in $\mathbb{F}$ (the diagram on left hand side below) whose image under $U$ is the pullback diagram on the right hand side below:
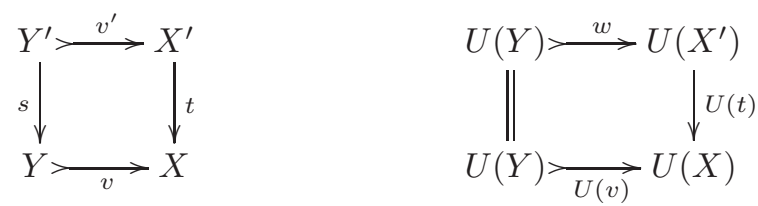

It easily follows that the map $v^{\prime}$ is $U$-cartesian above $w$.

Theorem 1.13. Let $\mathbb{E}$ be a pointed finitely complete category. Then it has normalizers if and only if the functor $K$ is fibrant on monomorphisms.

Proof. If the functor $K$ is fibrant on monomorphism, $\mathbb{E}$ has normalizers by Proposition 1.4. The converse is a consequence of Proposition 1.4. Lemma 1.12. and of the fact that any split extension can be embedded in some $J(T)$, see the following diagram:

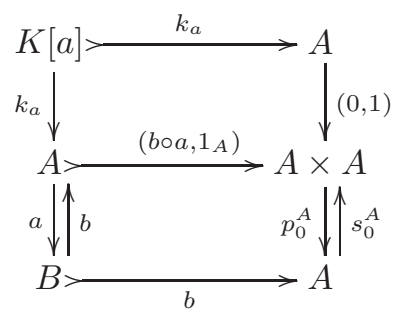

Choosing a kernel functor $K e r: P t \mathbb{E} \rightarrow \mathbb{E}$ is to specify an equivalence of category $K P t \mathbb{E} \simeq P t \mathbb{E}$, and saying that $K: K P t \mathbb{E} \rightarrow \mathbb{E}$ is fibrant on monomorphisms is equivalent to saying that $K e r: P t \mathbb{E} \rightarrow \mathbb{E}$ is quasi-fibrant on monomorphisms, namely that any monomorphism in $\mathbb{E}$ determines a $K e r$-cartesian map up to a unique isomorphism.

\section{Internal groups in $\mathbb{E}$}

In this section, we shall show that, when a category $\mathbb{E}$ is cartesian with finite limits, the category $G p \mathbb{E}$ of internal groups in $\mathbb{E}$ has normalizers. So let us suppose that $\mathbb{E}$ is cartesian closed; recall, see e.g. Lemma 1.5.2 in [18, that it is equivalent to saying that the pullback functor along any terminal map $\tau_{U}: U \rightarrow 1$ has a right adjoint; it implies, more generally, that the pullback functor along any projection $p_{T}: T \times U \rightarrow T$ has a right adjoint (denoted by $\left.\pi_{p_{T}}\right)$.

Now let $T$ be an internal group in $\mathbb{E}$. We shall denote by $\psi_{T}: T \times T \rightarrow T$ and $\tilde{\psi}_{T}: T \times T \rightarrow T$ the maps defined by the formulae $\psi_{T}(x, y)=x \circ y \circ x^{-1}$ and $\tilde{\psi}_{T}(x, y)=x^{-1} \circ y \circ x$. When $v: U \longmapsto T$ is any subobject, we denote by 
$\psi_{v}: T \times U \rightarrow T, \tilde{\psi}_{v}: T \times U \rightarrow T$ the restrictions $\psi_{v}(t, u)=t \circ u \circ t^{-1}$ and $\tilde{\psi}_{v}(t, u)=t^{-1} \circ u \circ t$. Let us denote by $c_{v}^{T}: C_{v}^{T} \longmapsto T \times U$ the inverse image of $v: U \longmapsto T$ along $\psi_{v}$ and $\tilde{c}_{v}^{T}: \tilde{C}_{v}^{T} \longmapsto T \times U$ the inverse image of $v$ along $\tilde{\psi}_{v}$. Let us consider now the projection $p_{T}: T \times U \rightarrow T$ and denote by $w_{v}: X_{v} \longmapsto T$ and $\tilde{w}_{v}: \tilde{X}_{v} \longmapsto T$ the subobjects $\pi_{p_{T}}\left(c_{v}^{T}\right)$ and $\pi_{p_{T}}\left(\tilde{c}_{v}^{T}\right)$.

Lemma 2.1. Let $\mathbb{E}$ be a cartesian closed category, $T$ an internal group and $v: U \longmapsto T$ any subobject. Then there is factorization $\psi$ :

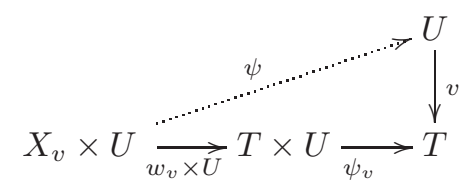

meaning that: $\forall(t, u) \in X_{v} \times T$, we have t.u.t $t^{-1} \in U$. Accordingly $X_{v}$ is a submonoid of $T$. The same holds for $\tilde{X}_{v}$, and the intersection $X=X_{v} \cap \tilde{X}_{v}$ is a subgroup of $T$.

Proof. Consider the following diagram where any square is a pullback:

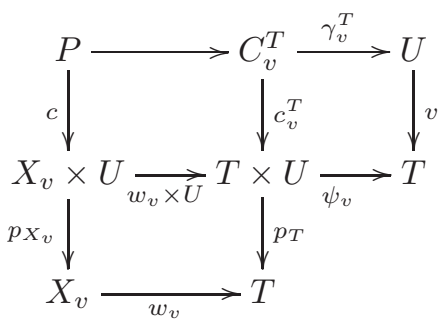

The Beck-Chevalley commutation associated with the lower left hand side pullback asserts that we have a natural isomorphism $w_{v}^{*} \cdot \pi_{p_{T}} \simeq \pi_{p_{X_{v}}} \cdot\left(w_{v} \times U\right)^{*}$. Since $w_{v}$ is a monomorphism, we have $w_{v}^{*} \cdot \pi_{p_{T}}\left(c_{v}^{T}\right)=w_{v}^{*}\left(w_{v}\right) \simeq 1_{X_{v}}$. Accordingly $\pi_{p_{X_{v}}}(c)=\pi_{p_{X_{v}}} \cdot\left(w_{v} \times U\right)^{*}\left(c_{v}^{T}\right)$ is an isomorphism. Now $c$ being a monomorphism, it is a subobject of the terminal object in $\mathbb{E} /\left(X_{v} \times U\right)$ whose image by the functor $\pi_{p_{X_{v}}}$ is the terminal object; the map $c$ is consequently itself an isomorphism and we get the desired factorization $\psi$.

Let us show now that $X_{v}$ is stable under the group operation of $T$. Let us denote it by $m: T \times T \rightarrow T$ and let us consider the following diagram:

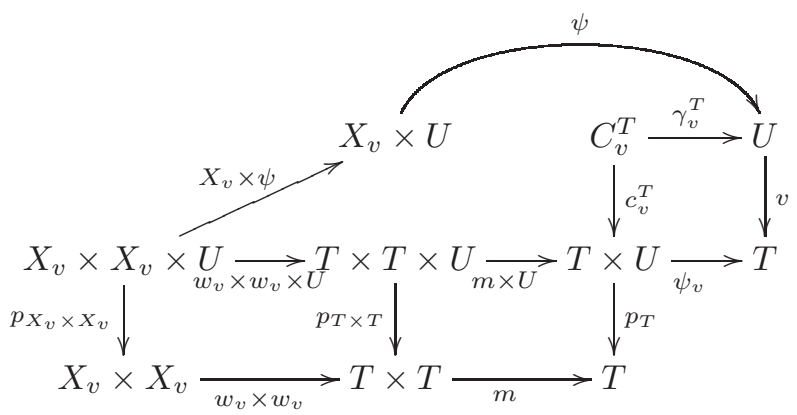


Since the right hand side lower square is a pullback, we get the Beck-Chevalley isomorphism $m^{*} \cdot \pi_{p_{T}} \simeq \pi_{p_{T \times T}} \cdot(m \times U)^{*}$. We are looking for a map from $w_{v} \times w_{v}$ to $m^{*}\left(w_{v}\right)=m^{*} \cdot \pi_{p_{T}}\left(c_{v}^{T}\right) \simeq \pi_{p_{T \times T}} \cdot(m \times U)^{*}\left(c_{v}^{T}\right)$ that is equivalent to a map from $p_{T \times T}^{*}\left(w_{v} \times w_{v}\right)=w_{v} \times w_{v} \times U$ to $(m \times U)^{*}\left(c_{v}^{T}\right)=(m \times U)^{*} \cdot \psi_{v}^{*}(v)$. It is produced by the map $\psi \cdot\left(X_{v} \times \psi\right)$ since we have $v \cdot \psi \cdot\left(X_{v} \times \psi\right)=\psi_{v} \cdot(m \times U) \cdot\left(w_{v} \times w_{v} \times U\right)$. So $X_{v}$ is a submonoid of $T$. The same proof holds for $\tilde{X}_{v}$ which becomes a submonoid as well. Accordingly $X=X_{v} \cap \tilde{X}_{v}$ is a submonoid of $T$ which, by definition, is stable under the passage to inverse; consequently it is a subgroup of $T$.

Proposition 2.2. Let $\mathbb{E}$ be cartesian closed category; then the category $G p \mathbb{E}$ of internal groups in $\mathbb{E}$ has normalizers.

Proof. Let $T$ be an internal group and, now, $v: U \longmapsto T$ any subgroup. Let us show that there is factorization: $u: U \longmapsto X=X_{v} \cap \tilde{X}_{v}$ such that $v=w . u$. Consider the following diagram where $\varepsilon$ is the universal arrow associated with $w_{v}=\pi_{p_{T}}\left(c_{v}^{T}\right)$ :

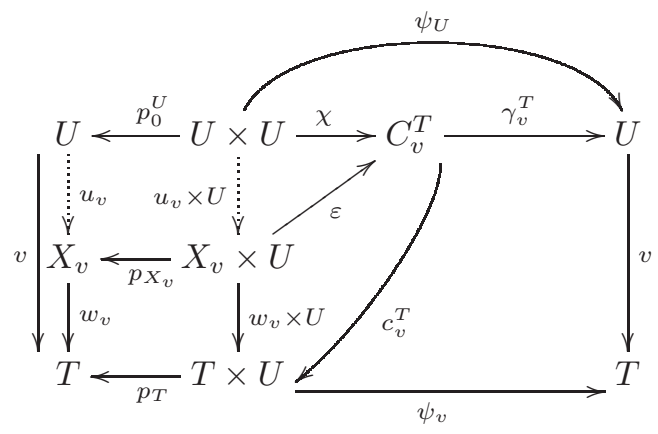

The identity $v \cdot \psi_{U}=\psi_{v} \cdot(v \times U)$ produces a factorization $\chi: U \times U \rightarrow C_{v}^{T}$ such that $c_{v}^{T} \cdot \chi=v \times U$ and $\gamma_{v}^{T} \cdot \chi=\psi_{U}$. By the universal property of $w_{v}=\pi_{p_{Y}}\left(c_{v}^{T}\right)$, this map $\chi$ produces a unique factorization $u_{v}: U \rightarrow X_{v}$ such that $v=w_{v} \cdot u_{v}$ and $\varepsilon \cdot\left(u_{v} \times U\right)=\chi$. This factorization is a map of monoids since so is $u$ and $w_{v}$ is a monomorphism. The same proof holds for the map $\tilde{w}_{v}: \tilde{X}_{v} \rightarrow T$ which leads to a factorization $v=\tilde{w}_{v} \cdot \tilde{u}_{v}$. Whence the factorization $v=w \cdot u$ with $w: X \longmapsto T$ a subgroup according to the previous lemma. By the Yoneda lemma a subgroup $u: U \longmapsto X$ is normal in $G p \mathbb{E}$ if and only if the map $\psi_{u}: X \times U \rightarrow X$ factors through $u$ (or equivalently $\tilde{\psi}_{u}: X \times U \rightarrow X$ factors through $u$ ). Accordingly, the monomorphism $u$ is normal, since the factorization map $\bar{\psi}: X \times U \rightarrow U$ given by the composite $X \times U \longmapsto X_{v} \times U \stackrel{\psi}{\rightarrow} U$ produces this factorization.

It remains to show that the universal property holds. So let $v=w^{\prime} \cdot u^{\prime}$ be a decomposition with $u^{\prime}$ a normal monomorphism. We are looking for a factorization from $w^{\prime}$ to $w=w_{v} \cap \tilde{w}_{v}=\pi_{p_{T}}\left(c_{v}^{T} \cap \tilde{c}_{v}^{T}\right)$ which is equivalent to a factorization from $p_{T}^{*}\left(w^{\prime}\right)=w^{\prime} \times U$ to $c_{v}^{T} \cap \tilde{c}_{v}^{T}$. We get a factorization from 
$w^{\prime} \times U$ to $c_{v}^{T}$ by the dotted arrow in following diagram:

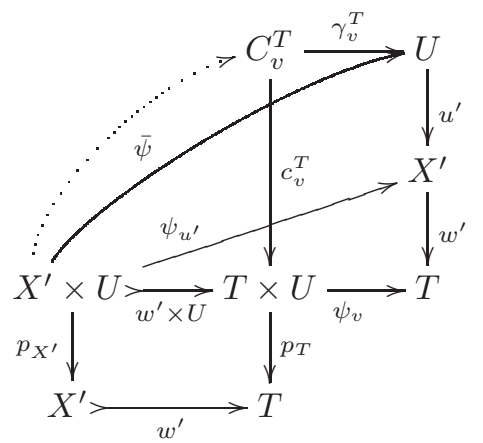

which is produced by the factorization $\bar{\psi}$ given by the normal monomorphism $u^{\prime}$; and we get a factorization from $w^{\prime} \times U$ to $\tilde{c}_{v}^{T}$ by the dotted arrow in following diagram:

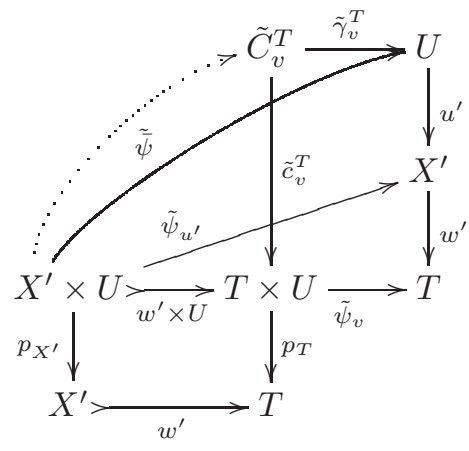

produced by the factorization $\tilde{\bar{\psi}}$ given by the normal monomorphism $u^{\prime}$

Corollary 2.3. Let $\mathbb{E}$ be a locally cartesian closed category. For each object $B$ in $\mathbb{E}$, the category $G p(\mathbb{E} / B)=G p\left(P t_{B} \mathbb{E}\right)$ has normalizers.

For regular cartesian closed categories, the existence of normalizers can be proved another way. In [3] it was shown that for any cartesian closed category $\mathbb{D}$, each split extension functor in the category $G p \mathbb{D}$ is representable; and in [17] it was shown that for a protomodular category $\mathbb{C}$, the category $\mathbb{C}$ has normalizers whenever each split extension functor in $\mathbb{C}^{2}$ is representable. However even when $\mathbb{C}$ is protomodular the normalizers considered there have a different universal property, they were universal amongst those factorizations where the first morphism is a normal monomorphism (in the sense of being a kernel of some morphism) and the second is a monomorphism. These two definitions coincide when the category is exact protomodular. It can be checked that replacing normal monomorphism (in sense of being a kernel) with normal monomorphism (in sense of Definition 1.1), the proof of the implications $(c) \Rightarrow(b) \Rightarrow(a)$ in Theorem 3.7 lifts. We have: 
Proposition 2.4. Let $\mathcal{V}$ be any semi-abelian variety of universal algebras such that for any cartesian closed category $\mathbb{D}$, with finite limits, each split extension functor in the category $\mathcal{V}(\mathbb{D})$ of internal such algebras in $\mathbb{D}$, is representable. For each finitely complete regular cartesian closed category $\mathbb{E}$ the category $\mathcal{V}(\mathbb{E})$ has normalizers.

Proof. It is easy to check that if $\mathbb{E}$ is a regular category, then $\mathcal{V}(\mathbb{E})$ is a regular category, and it is certainly a Mal'tsev category. Since in regular Mal'tsev categories normal monomorphisms are stable under regular images [7] it follows that we need only show that the normalizer of monomorphism is terminal amongst those factorizations where the second morphism is a monomorphism. Since $\mathbb{E}$ is cartesian closed and has finite limits, it follows that the category of maps $\mathbb{E}^{2}$ is cartesian closed, and so by assumption each split extension functor in the category $\mathcal{V}\left(\mathbb{E}^{2}\right)$ is representable. Since $\mathcal{V}\left(\mathbb{E}^{2}\right) \cong \mathcal{V}(\mathbb{E})^{2}$ it follows from remarks preceding the proposition that $\mathcal{V}(\mathbb{E})$ has normalizers.

Remark 2.5. In addition to the category of groups, the category of Lie algebras (over a fixed commutative ring) satisfies the assumptions of Proposition 2.4 [3] and so for each finitely complete regular cartesian closed category $\mathbb{E}$ the categories of internal such algebras in $\mathbb{E}$ have normalizers.

\section{Action accessibility}

In this section we shall show that any exact protomodular category with normalizers is action accessible in the sense of [10].

\subsection{Action distinctive categories}

Recall from [6] that a Mal'tsev category $\mathbb{C}$ has centralizers of equivalence relations if and only if it is action distinctive, namely if and only if for any object $(f, s)$ in $P t \mathbb{C}$, there is a greatest $\mathrm{P}_{\mathbb{C}}$-cartesian equivalence relation $\mathbb{D}[f, s]$ on $(f, s)$ called its distinctive equivalence relation and denoted by

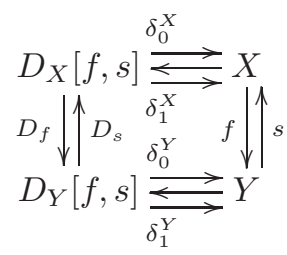


The centralizer of an equivalence relation $R$ on $X$ is then given by the lower level of the distinctive equivalence relation of the split epimorphism $\left(d_{0}, s_{0}\right): R \rightleftarrows X$ :

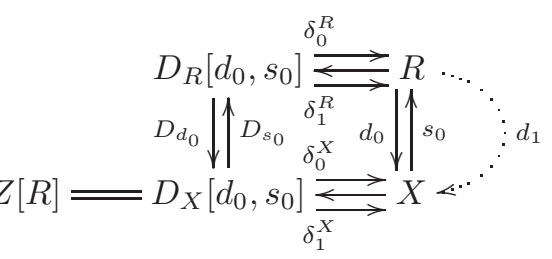

Lemma 3.1. Let $\mathbb{C}$ be any pointed action distinctive Mal'tsev category. Then the distinctive equivalence relation of $\left(p_{T},(1,0)\right): T \times X \rightleftarrows T$ is the following one:

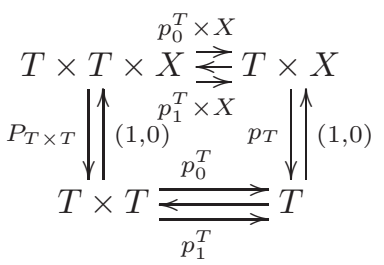

Proof. It is clearly a $\mathrm{P}_{\mathbb{C}^{-} \text {-cartesian equivalence relation since each of the com- }}$ muting squares above is a pullback, and

$$
T \times T \underset{p_{1}}{\stackrel{p_{0}}{\rightleftarrows}} T
$$

is the largest possible equivalence relation at the lower level.

The Mal'tsev category $\mathbb{C}$ is said to be functorially action distinctive when in addition there is a functorial extension of $\mathbb{D}$ to the $\mathrm{P}_{\mathbb{C}}$-cartesian maps; this property is the common part of action accessible and pointed B-C facc categories, as are $G p, R g, R$-Lie and TopGp, see [6]; it implies that any fibre $P t_{Y} \mathbb{C}$ has centralizers of equivalence relations and that any change of base functor with respect to $\mathrm{P}_{\mathbb{C}}$ preserves the centralizers of equivalence relations. Now we get the following:

Proposition 3.2. Let $\mathbb{C}$ be a pointed protomodular category with normalizers. Then it is functorially action distinctive.

Proof. Let $(f, s)$ be a split epimorphism and consider the following right hand side $K$-cartesian map above the diagonal $s_{0}: K[f] \longmapsto K[f] \times K[f]$ :

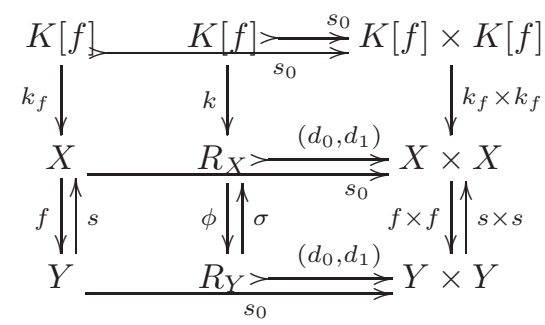


it determines relations $R_{X}$ on $X$ and $R_{Y}$ on $Y$ which, actually, produce a relation in $P t \mathbb{C}$ on $(f, s)$. The factorization of the map between the extremal split extensions in the diagram above through this $K$-cartesian map is described below and shows that both relations are reflexive, and thus equivalence relations:

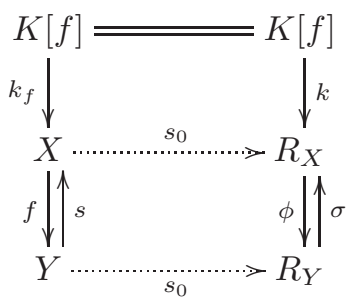

Moreover, since $\mathbb{C}$ is protomodular, the lower square is a pullback and this becomes an equivalence relation in $P t \mathbb{C}$ on $(f, s)$ whose two legs are $\mathrm{P}_{\mathbb{C}}$-cartesian maps. It is easy to show that it is the action distinctive equivalence relation associated with $(f, s)$ : take another equivalence relation $S$ in $\operatorname{Pt} \mathbb{C}$ on $(f, s)$ whose two legs are $\mathrm{P}_{\mathbb{C}}$-cartesian maps. Now this last fact makes the left hand side a split extension and produces the following map between the two extremal split extensions:

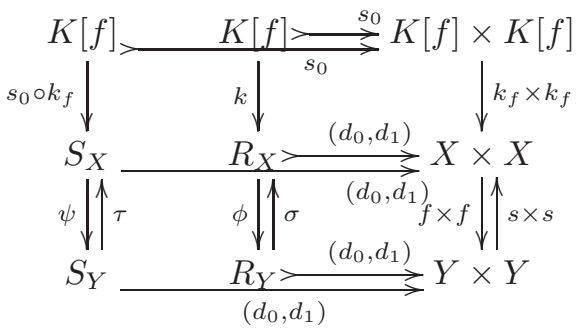

Its factorization $S \rightarrow R$ through the right hand side $K$-cartesian map makes $R$ the distinctive equivalence relation on $(f, s)$. Accordingly $\mathbb{C}$ is action distinctive. The fact that it is functorially action distinctive is a consequence of Proposition 1.5

\subsection{Eccentric and faithful split epimorphisms}

When $\mathbb{C}$ is an action distinctive Mal'tsev category, a split epimorphism $(f, s)$ : $X \rightleftarrows Y$ of $P t \mathbb{C}$ is said to be eccentric when the distinctive equivalence relation $\mathbb{D}[f, s]$ is the discrete one, which is the case if and only if $s^{-1}(Z[R[f]])=\Delta_{Y}$. An object $(f, s)$ is said to be faithful when, given any split epimorphism $\left(f^{\prime}, s^{\prime}\right)$ 
and any pair of parallel $\mathrm{P}_{\mathbb{C}^{-}}$-artesian maps:

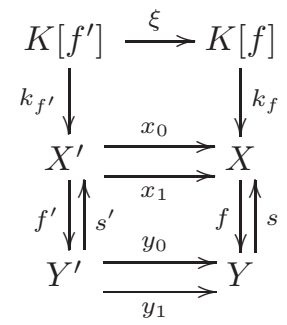

they are equal as soon as they induce the same (iso-)morphism $\xi$ at the level of their kernels. Any faithful split epimorphism $(f, s)$ is eccentric: the two legs of its distinctive equivalence relation produce a pair of parallel $\mathrm{P}_{\mathbb{C}^{-}}$-cartesian maps with codomain $(f, s)$; being jointly split, these maps induce the same isomorphism at the level of their kernels. Accordingly they are equal, and the distinctive equivalence relation is discrete.

Proposition 3.3. Let $\mathbb{C}$ be a pointed protomodular category with normalizers. Then a split epimorphism is eccentric if and only if it is faithful.

Proof. Since $(f, s)$ is eccentric and $\mathbb{C}$ has normalizers, the following right hand side vertical diagram is a $K$-cartesian map according to Proposition 3.2

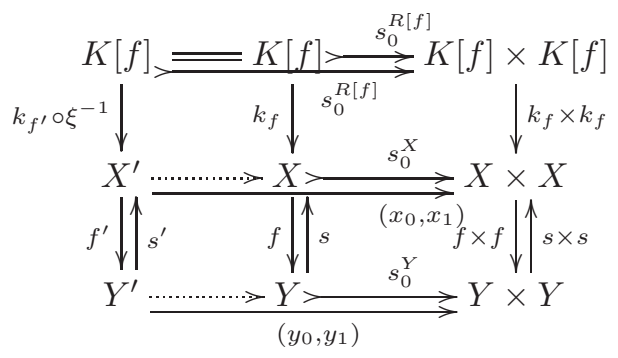

Let $\left(x_{0}, y_{0}\right):\left(f^{\prime}, s^{\prime}\right) \rightarrow(f, s)$ and $\left(x_{1}, y_{1}\right):\left(f^{\prime}, s^{\prime}\right) \rightarrow(f, s)$ be a parallel pair of maps of split epimorphisms producing the same factorization $\xi$. In the diagram above, the universal property of the $K$-cartesian map produces a dotted factorization which means that $x_{0}=x_{1}$ and $y_{0}=y_{1}$; accordingly $(f, s)$ is faithful.

A split epimorphism $(f, s): X \rightleftarrows Y$ in the category $G p$ of groups is faithful if and only if the associated group homomorphism $Y \longmapsto A u t(K e r f)$ is injective. Similarly, it is faithful in a category $R$-Lie of Lie algebras (for some ring $R$ ) if and only if the associated homomorphism $Y \longmapsto \operatorname{Der}(\operatorname{Ker} f)$ is injective.

\subsection{Action accessibility}

Recall that a Mal'tsev category $\mathbb{C}$ is action accessible 10 if the category $\operatorname{Pt} \mathbb{C}$ has enough faithful objects, namely if, for any split epimorphism $(f, s)$, there is a $\mathrm{P}_{\mathbb{C}^{-} \text {cartesian map }}(f, s) \rightarrow(g, t)$ whose codomain is faithful. The categories 
$G p$ of groups, $R g$ of non commutative rings, $R$-Lie of Lie algebras and TopGp of topological groups are examples of this notion. Now we get:

Proposition 3.4. When $\mathbb{C}$ is an exact action distinctive Mal'tsev category, it has enough eccentric objects. When $\mathbb{C}$ is an exact pointed protomodular category with normalizers, it is action accessible.

Proof. Let $(f, s)$ be a split epimorphism. Consider its distinctive equivalence relation:

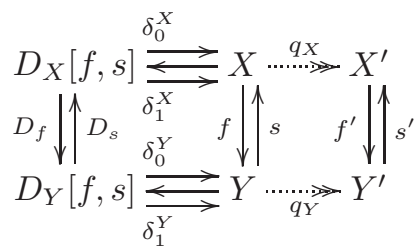

Complete the diagram by the quotients of the level-wise equivalence relations; since pulling back along split epimorphisms reflects isomorphisms, the right hand side downward square is a pullback since so are the left hand side ones. Accord-

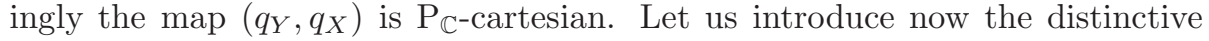
equivalence relation $\mathbb{D}\left[f^{\prime}, s^{\prime}\right]$. In the exact context the distinctive equivalence relations have a functorial extension on the regular epimorphisms (Proposition 5.3 in [6]). Accordingly we have $\left(q_{Y}, q_{X}\right)^{-1}\left(\Delta_{\left(f^{\prime}, s^{\prime}\right)}\right)=R\left[q_{Y}, q_{X}\right]=\mathbb{D}[f, s]=$ $\left(q_{Y}, q_{X}\right)^{-1}\left(\mathbb{D}\left[f^{\prime}, s^{\prime}\right]\right)$. Thus we get $\mathbb{D}\left[f^{\prime}, s^{\prime}\right]=\Delta_{\left(f^{\prime}, s^{\prime}\right)}$, and $\left(f^{\prime}, s^{\prime}\right)$ is eccentric. When moreover $\mathbb{C}$ is exact and pointed protomodular with normalizers, $\left(f^{\prime}, s^{\prime}\right)$ is faithful according to the previous proposition.

The construction above is precisely the way that the category $R g$ of non commutative rings was proved to be action accessible in [10, since the ideals used in this proof were precisely the ideals associated with the equivalence relations $D_{Y}[f, s]$ and $D_{X}[f, s]$ given above.

\section{B-C facc categories}

In this section we shall show that any pointed protomodular category $\mathbb{C}$ with normalizers is B-C facc. First recall that a category $\mathbb{C}$ is unital when it is pointed, finitely complete and such that the following pair is jointly strongly epic:

$$
X \stackrel{(1,0)}{\longrightarrow} X \times Y \stackrel{(0,1)}{\longrightarrow} Y
$$

Any pointed protomodular category is unital. In a unital category there is an intrinsic notion of commuting pair of maps having same codomain.

\subsection{Centralizers of normal subobjects}

Let us begin with the following: 
Lemma 4.1. Let $\mathbb{C}$ be a unital category. Suppose the monomorphism $u: U \mapsto$ $X$ is normal to an equivalence relation $R$ on $X$ and $t: T \rightarrow X$ is any map. The pair $(u, t)$ commutes in $\mathbb{C}$ if and only if there is a (unique) map $\psi$ making the following diagram a map of split extensions:

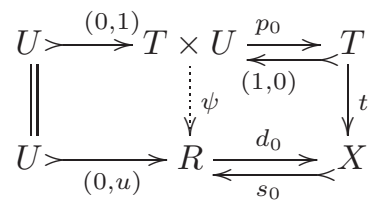

Proof. Suppose the map $\psi$ exists, then the map $\phi=d_{1} \circ \psi: T \times U \rightarrow X$ is the desired cooperator of the pair $(u, t)$ since we have: $d_{1} \circ \psi \circ(0,1)=d_{1} \circ(0, u)=u$ and $d_{1} \circ \psi \circ(1,0)=d_{1} \circ s_{0} \circ t=t$. Conversely let $\phi: T \times U \rightarrow X$ be the cooperator of the pair $(u, t)$. Since $(1,0)$ and $(0,1)$ are jointly strongly epimorphic, on the one hand we can check that the diagram

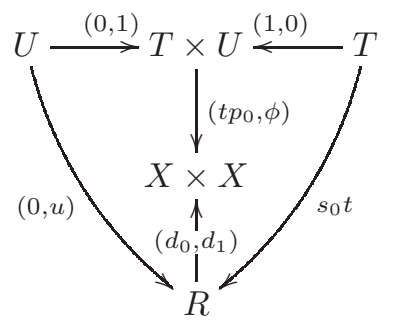

commutes; and on the other hand, $\left(d_{0}, d_{1}\right)$ being a monomorphism, it follows that there exists a map $\psi: T \times U \rightarrow R$ with $\psi(0,1)=(0, u)$ and $\psi(1,0)=s_{0} t$. The proof is completed by noting that, again since $(1,0)$ and $(0,1)$ are jointly epimorphic, we have trivially $d_{0} \psi=t p_{0}$.

The following proposition extends an observation of 14 from action accessible categories to any functorially action distinctive category:

Proposition 4.2. Let $\mathbb{C}$ be a pointed protomodular category which is functorially action distinctive. Suppose the monomorphism $u: U \longmapsto X$ is normal to an equivalence relation $R$ on $X$, and denote by $Z[R]$ the centralizer of the equivalence relation $R$. Then the normalization of the equivalence relation $Z[R]$ is the centralizer of the monomorphism $u$. In other words, any normal subobject has a centralizer which is normal.

Proof. Let $t: T \rightarrow X$ be any map commuting with $u$. Since any pointed protomodular category is unital, according to Lemma 4.1 there is a map $\psi$ making the following diagram commute:

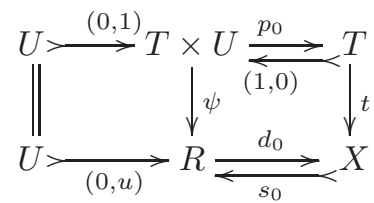


Since the row are split extension and the pointed category $\mathbb{C}$ is protomodular, the right hand side square is a pullback which determines a $\mathrm{P}_{\mathbb{C}^{-}}$-cartesian in $P t \mathbb{C}$. Since moreover $\mathbb{C}$ is functorially action distinctive, then $t^{-1}(Z[R])=$ $D_{T}\left(p_{T},(1,0)\right)=\nabla_{T}$, according to Lemma 3.1. Then the following diagram asserts that the map $t$ factors through the normalization $\nu$ of $Z[R]$ which consequently becomes the earnest centralizer of $u$ :

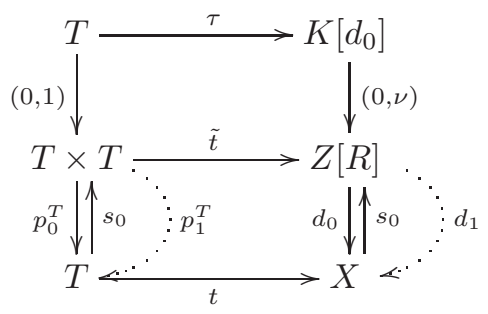

since we have $\nu \circ \tau=d_{1} \circ(0, \nu) \circ \tau=t \circ p_{1}^{T} \circ(0,1)=t$.

\subsection{Algebraically cartesian closed categories}

A unital category is said to be algebraically cartesian closed (acc) (see [9], originally considered in [15, 16]) when the pullback functors along the terminal maps between categories of points have right adjoints. This was shown in 9] to be equivalent to any subobject having an earnest centralizer (that is a universal map commuting with it; cf. Definition 3.2.2 [15]; this map is necessarily a monomorphism).

Proposition 4.3. Let $\mathbb{C}$ be a unital category with normalizers. Then any map commuting with $v$ factors through the normalizer of $v$. If, in addition, $\mathbb{C}$ has earnest centralizers of normal subobjects. Then it is acc.

Proof. (cf. Proposition 2.1 in [17]) Let $v: U \longmapsto T$ be any monomorphism and $u: U \longmapsto X$ its normalizer. Let $t: \bar{T} \rightarrow T$ be any map commuting with $v$ and $\phi: U \times \bar{T} \rightarrow T$ the cooperator of this pair:

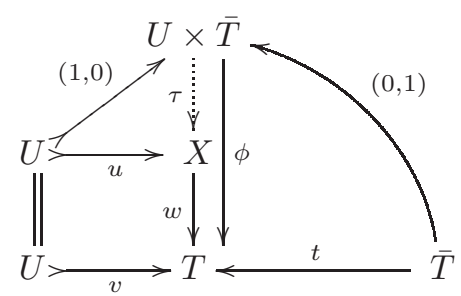

Then we have $\phi \circ(1,0)=v$ where $(1,0)$ is a normal monomorphism. Now since $u$ is the normalizer of $v$, we get a factorization $\tau: U \times \bar{T} \longmapsto X$ such that $\tau \circ(1,0)=u, w \circ \tau=\phi$ and consequently $w \circ \tau .(0,1)=t$. Moreover the pair $(u, \tau \circ(0,1))$ commutes since, composed with the monomorphism $w$, it gives rise to the commuting pair $(v, t)$. 
Suppose that $u$ has an earnest centralizer $\nu: Z[u] \longmapsto X$, there is a factorization $\xi: \bar{T} \rightarrow Z[u]$ such that $\nu \circ \xi=\tau \circ(0,1)$ and consequently the following identity: $(w \circ \nu) \circ \xi=w \circ \tau \circ(0,1)=\phi \circ(0,1)=t$ produces the required factorization which show that $Z[u] \stackrel{\nu}{\longmapsto} X \stackrel{w}{\longmapsto} T$ is the earnest centralizer of $v$.

Corollary 4.4. Let $\mathbb{C}$ be a pointed protomodular category with normalizers. Then it is acc.

Proof. The category $\mathbb{C}$ is functorially action distinctive by proposition 3.2 According to the Proposition 4.2 any normal monomorphism has an earnest centralizer. Any pointed protomodular category being unital, the conclusion comes from Proposition 4.3 ,

It is worth describing a direct construction of the earnest centralizer of a monomorphism $v: U \longmapsto T$. For that consider the normalizing decomposition $U \stackrel{n}{\longmapsto} S \stackrel{\left(r_{0}, r_{1}\right)}{\longmapsto} T \times T$ of the monomorphism $(v, v): U \longmapsto T \times T$. Now consider the following pullback:

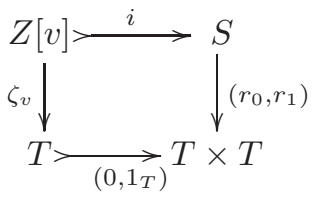

The monomorphism $i$ is normal since $\left(0,1_{T}\right)$ is normal.

Proposition 4.5. Let $\mathbb{C}$ be a pointed protomodular category with normalizers. Then the monomorphism $\zeta_{v}$ is the earnest centralizer of $v$. When $v$ is normal, $S$ is an equivalence relation and $\zeta_{v}$ the normalization of this equivalence relation.

Proof. The following rectangle is a pullback since $v$ is a monomorphism:

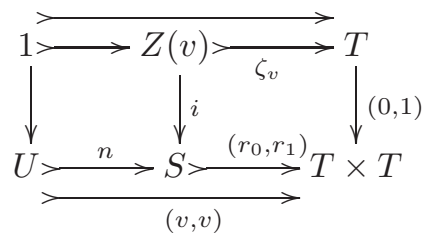

Accordingly, since the right hand side square is a pullback, the left hand square is a pullback and shows that the intersection of the two normal monomorphisms $n$ and $i$ is trivial. Accordingly, since the category $\mathbb{C}$ is pointed protomodular, these two normal monomorphisms do commute, see [4. As a consequence, the subobjects $v=r_{1} \circ n$ and $\zeta_{v}=r_{1} \circ i$ commute. Let $t: \bar{T} \rightarrow T$ be any map commuting with $v$. Then the composite $(0,1) \circ t: \bar{T} \rightarrow T \times T$ commute with $(v, v)$ and, according to Proposition 4.3 , factors through $S$, and thus $t$ factors through $Z[v]$. When $v$ itself is normal, the decomposition $(v, v)=s_{0}^{T} \circ v$ produces a factorization $T \rightarrow S$ which makes $S$ a reflexive relation and thus an equivalence relation. 


\subsection{Fibrewise algebraically cartesian closed categories}

A fibrewise algebraically cartesian closed category (facc) Mal'tsev category [9] is a Mal'tsev category $\mathbb{C}$ whose any fibre $P t_{Y} \mathbb{C}$ is algebraically cartesian closed which is equivalent to saying that any fibre $P t_{Y} \mathbb{C}$ has centralizers of subobjects. It is called B-C facc [6] when in addition any change of base functor with respect to the fibration $\mathrm{P}_{\mathbb{C}}$ preserves those centralizers; we recalled that any pointed B-C facc Mal'tsev category is functorially action distinctive. Although the definition of a normalizer is not quite the same here, we omit the proof of the next proposition since its proof is essentially the same as the proof of Proposition 1.13 in [17].

Proposition 4.6. Let $\mathbb{C}$ be any pointed protomodular category. Suppose it has normalizers. Then any fibre $P t_{T} \mathbb{C}$ has normalizers. Moreover any change of base functor with respect to the fibration $\mathrm{P}_{\mathbb{C}}$ preserves the normalizers.

Whence the following straightforward consequence:

Theorem 4.7. Let $\mathbb{C}$ be any pointed protomodular category. When it has normalizers, it is $B$-C facc.

Proof. According to the previous proposition, since $\mathbb{C}$ has normalizers, the same holds for the pointed protomodular fibre $P t_{Y} \mathbb{C}$. By Corollary 4.4, any fibre $P t_{Y} \mathbb{C}$ is then acc, and $\mathbb{C}$ is facc. Moreover, by the previous proposition any change of base functor with respect to $\mathrm{P}_{\mathbb{C}}$ preserves the normalizers. We recalled above that these change of base functors preserve the centralizers of equivalence relations in the fibre $P t_{Y} \mathbb{C}[6]$ and consequently, in the protomodular context, the centralizers of normal subobjects (see Proposition 4.2) of these fibres. Consequently, following the construction given in Proposition 4.3, it preserves any centralizer, and the category $\mathbb{C}$ is $\mathrm{B}-\mathrm{C}$ facc.

This result could be understood as a partial algebraic counterpart of the cartesian closedness assumed in Section 2, since a category is facc if and only if the change of base functor with respect to the fibration $\mathrm{P}_{\mathbb{C}}$ along any split epimorphism (resp. any regular epimorphism in the regular context) has a right adjoint.

\section{References}

[1] M. Barr, Exact categories, Springer L.N. in Math., 236, 1971, 1-120.

[2] F. Borceux and M.M. Clementino, Topological semi-abelian algebras, Advances in Mathematics, 190, 2005, 425-453.

[3] F. Borceux, G. Janelidze, and G. M. Kelly, On the representability of actions in a semi-abelian category, Theory and Applications of Categories, 14, 2005, 244-286. 
[4] D. Bourn, Normal subobjects and abelian objects in protomodular categories, Journal of Algebra, 228, 2000, 143-164.

[5] D. Bourn, Normal subobjects of topological groups and of topological semiAbelian algebras, Topology and its applications, 153, 2006, 1341-1364

[6] D. Bourn, Two ways to centralizers of equivalence relations, preprint, 2012.

[7] D. Bourn and F. Borceux, Mal'cev, Protomodular, Homological and SemiAbelian Categories, Kluwer, Mathematics and Its Applications, vol. 566, 2004, 479 pp.

[8] D. Bourn and M. Gran, Centrality and normality in protomodular categories, Theory and Applications of Categories, 9, 2002, 151-165.

[9] D. Bourn and J.R.A. Gray, Aspects of algebraic exponentiation, Bull. Belg. Math. Soc. Simon Stevin, 19, 2012, 823-846.

[10] D. Bourn and G. Janelidze Centralizers in action accessible categories, Cahiers de Top. et Géom. Diff. Catégoriques, 50, 2009, 211-232.

[11] D. Bourn and Z. Janelidze, Categorical (binary) difference terms and protomodularity, Algebra Universalis, Algebra universalis, 66, 2011, 277-316.

[12] A. Carboni, J. Lambek and M.C. Pedicchio, Diagram chasing in Mal'cev categories, J. Pure Appl. Algebra, 69, 1991, 271-284.

[13] A. Carboni, M.C. Pedicchio and N. Pirovano, Internal graphs and internal groupoids in Mal'cev categories, CMS Conference Proceedings, 13, 1992, 97-109.

[14] A. Cigoli and S. Mantovani, Action accessibility and centralizers, J. Pure Appl. Algebra, 216, 2012, 1852-1865.

[15] J.R. A. Gray, Algebraic exponentiation and internal homology in general categories, $\mathrm{PhD}$ thesis, University of Cape Town, 2010.

[16] J.R. A. Gray, Algebraic exponentiation in general categories, Applied Categorical Structures, 20, 2012, 543-567.

[17] J.R.A. Gray, Normalizers, centralizers and action representability in semiabelian categories, preprint, 2012.

[18] P.T. Johnstone, Sketches of an Elephant: a Topos Theory Compendium, Oxford University Press, Oxford 2002.

[19] S. Lang, Algebra, Addison-Wesley Publishing Company, 1965.

MSC (AMS classification): 18A05; 18B99; 08C05; 08A30; 08A99; 22A05

Keywords: Categorical algebra; Algebraic theory; Normalizer; Split extension; Fibration of points, Protomodular category; Mal'tsev category; Unital category; Topological algebra 